\title{
ISWI and CHD Chromatin Remodelers Bind Promoters but Act in Gene Bodies
}

\author{
Gabriel E. Zentner ${ }^{1}$, Toshio Tsukiyama ${ }^{1}$, Steven Henikoff ${ }^{1,2 *}$
}

1 Basic Sciences Division, Fred Hutchinson Cancer Research Center, Seattle, Washington, United States of America, 2 Howard Hughes Medical Institute, Fred Hutchinson Cancer Research Center, Seattle, Washington, United States of America

\begin{abstract}
ATP-dependent nucleosome remodelers influence genetic processes by altering nucleosome occupancy, positioning, and composition. In vitro, Saccharomyces cerevisiae ISWI and CHD remodelers require $\sim 30-85$ bp of extranucleosomal DNA to reposition nucleosomes, but linker DNA in S. cerevisiae averages $<20 \mathrm{bp}$. To address this discrepancy between in vitro and in vivo observations, we have mapped the genomic distributions of the yeast Isw1, Isw2, and Chd1 remodelers at base-pair resolution on native chromatin. Although these remodelers act in gene bodies, we find that they are also highly enriched at nucleosome-depleted regions (NDRs), where they bind to extended regions of DNA adjacent to particular transcription factors. Surprisingly, catalytically inactive remodelers show similar binding patterns. We find that remodeler occupancy at NDRs and gene bodies is associated with nucleosome turnover and transcriptional elongation rate, suggesting that remodelers act on regions of transient nucleosome unwrapping or depletion within gene bodies subsequent to transcriptional elongation.
\end{abstract}

Citation: Zentner GE, Tsukiyama T, Henikoff S (2013) ISWI and CHD Chromatin Remodelers Bind Promoters but Act in Gene Bodies. PLoS Genet 9(2): e1003317. doi:10.1371/journal.pgen.1003317

Editor: Gregory P. Copenhaver, The University of North Carolina at Chapel Hill, United States of America

Received October 18, 2012; Accepted December 27, 2012; Published February 28, 2013

Copyright: (C) 2013 Zentner et al. This is an open-access article distributed under the terms of the Creative Commons Attribution License, which permits unrestricted use, distribution, and reproduction in any medium, provided the original author and source are credited.

Funding: This work was supported by funding from the NIH (5U01 HG004274,U54 CA143862, R01 ES020116) and the Howard Hughes Medical Institute. The funders had no role in study design, data collection and analysis, decision to publish, or preparation of the manuscript.

Competing Interests: The authors have declared that no competing interests exist.

*E-mail: steveh@fhcrc.org

\section{Introduction}

Nucleosome remodelers use the energy of ATP hydrolysis to alter histone-DNA contacts, slide nucleosomes, and exchange or remove histones and entire nucleosomes. They are ubiquitous throughout eukaryotic evolution [1], and alterations in their expression are found in human congenital anomaly syndromes and cancers [2-5], highlighting their central role in cellular life.

Yeast Isw1, Isw2, and Chdl remodeler complexes bind $\sim 30$ $85 \mathrm{bp}$ of extranucleosomal DNA, which is required for efficient nucleosome repositioning in vitro [6-10], and histone H3 depletion stimulates Isw2 nucleosome sliding in vivo [11], presumably by creating regions of accessible DNA. Additionally, the human ISWI remodeler SNF2h functions in vitro as a dimeric sensor of linker length, with one ATPase molecule contacting extranucleosomal DNA on each side of a nucleosome, and the efficiency of its nucleosome repositioning activity is decreased with decreasing linker length [12-14]. Similarly, the human CHD7 remodeler, mutated in the developmental disorder CHARGE syndrome [5], requires $\geq 40$ bp of extranucleosomal DNA for remodeling in vitro [15]. Yeast Isw1, Isw2, and Chdl act at various points in gene bodies [16-21], which contain arrays of regularly spaced nucleosomes [22]. However, the average nucleosomal repeat length in $S$. cerevisiae is 165 bp [22]. Given that 147 bp of DNA is wrapped around the histone octamer, only $\sim 18 \mathrm{bp}$ of DNA is extranucleosomal. Thus, much more DNA on either side of a nucleosome is required for ISWI and CHD proteins to act than is available between nucleosomes. This paradox is even more severe in $S$. pombe, where the Chdl-like remodelers hrpl and hrp3 act within gene bodies [23-25], but nucleosomal repeats average $154 \mathrm{bp}$ and linkers are therefore only $\sim 7$ bp [26].

To address this paradox, we mapped the genomic distributions of yeast Isw1, Isw2, and Chd1 on native chromatin. We show that yeast ISWI and CHD remodelers are highly enriched at nucleosome-depleted regions (NDRs) flanking transcription units, where they bind to extended stretches of linker DNA flanking transcription factor binding sites (TFBSs). We find that remodeler binding is positively associated with nucleosome turnover and transcriptional elongation rate, suggesting that ISWI and CHD remodelers first associate with naked DNA within NDRs and subsequently relocate to gene bodies following nucleosome disruption by RNA Polymerase II transit, upon which there is ample linker DNA to promote their efficient remodeling activity.

\section{Results/Discussion}

Genome-wide mapping of remodeler binding on native chromatin

We performed immunoprecipitation of uncrosslinked (native) chromatin digested with micrococcal nuclease (MNase) combined with high-throughput paired-end sequencing (N-ChIP-seq) for $S$. cerevisiae Isw1, Isw2, and Chd1. N-ChIP does not rely on formaldehyde fixation, which crosslinks primary amines such as those in lysine-rich histones [27]. Thus, we circumvent crosslinking of remodelers solely to their nearest nucleosome [17] and are able to directly map the interaction of remodelers with DNA. We and others have previously mapped transcription factors ( $\mathrm{TFs}$ ) on native chromatin from yeast and human cells [28-30], and we have shown 


\section{Author Summary}

Eukaryotic genomes are compacted into chromatin, which restricts access to DNA. In order for cells to transcribe, replicate, and repair DNA, chromatin structure must be altered. Eukaryotes have evolved chromatin remodeling enzymes that use energy derived from ATP hydrolysis to modulate chromatin structure. In vitro, yeast ISWI and CHD remodelers require $30-85$ bp of extranucleosomal DNA in order to efficiently remodel chromatin, but in vivo, yeast linker DNA is, on average, $<20$ bp. By mapping yeast Isw1, Isw2, and Chd1 on native chromatin, we find that these remodelers bind to extended regions of linker DNA adjacent to transcription factor binding sites within nucleosome depleted regions. Remodeler binding is associated with nucleosome turnover and transcription rate, suggesting that ISWI and CHD remodelers help to reestablish proper chromatin structure following transcriptional elongation.

similar recovery of MNase-protected DNA fragments from both total nuclei and solubilized chromatin from yeast without crosslinking [30]. Thus, it appears that formaldehyde crosslinking may be dispensable for the detection of protein-DNA interactions.

ChIP and input samples were prepared for sequencing using a modified protocol that recovers fragments as small as $\sim 25 \mathrm{bp}$, enabling base-pair resolution mapping of remodeler occupancy [30]. Samples treated with MNase for 2.5' displayed a nucleosomal peak centered at $\sim 170 \mathrm{bp}$, which was shifted to $\sim 160 \mathrm{bp}$ in the $10^{\prime}$ samples (Figure 1A). Examination of these size distributions indicated that ChIP samples were enriched for supernucleosomal DNA fragments relative to nucleosomes. To assess this observation systematically, we determined the area under the curve for nucleosomal (141$250 \mathrm{bp}$ ) and supernucleosomal (251-428 bp) fragments from each sample. ChIP samples, regardless of factor, displayed supernucleoso$\mathrm{mal} /$ nucleosomal ratios 1.74-3.87 (2.5' MNase, $P=0.009)$ and 2.26$2.76\left(10^{\prime}\right.$ MNase, $\left.P=0.002\right)$ times greater than that seen in input samples (Table S2), suggesting that Isw1, Isw2, and Chd1 participate in the protection of large stretches of DNA.

N-ChIP-seq revealed specific sites of enrichment for all three remodelers throughout the yeast genome (Figure 1B and Figure $\mathrm{S} 1)$. We then tested if we could recapitulate known features of remodeler-genome association by $\mathrm{N}-\mathrm{ChIP}$-seq. We focused on Isw2, which has previously been characterized by genome-wide ChIP-chip [20,31] and the recently developed ChIP-exo method [17]. ChIP-exo is a modification of the standard ChIP-seq protocol employing exonuclease digestion to improve the resolution of crosslinking ChIP-seq. Our N-ChIP-seq methodology is distinct from these techniques in that it does not rely on formaldehyde fixation, which also crosslinks primary amines to fix protein-protein interactions. We first obtained lists of Isw2 peaks determined by ChIP-chip [20] and ChIP-exo [17] as well as sites with altered nucleosome positioning in an isw2 24 strain [20]. We then determined the input-normalized N-ChIP-seq signal for each base pair in a 2-kb window centered on each ChIP or remodeling site and averaged the signal for each base pair within the window for each class of sites (Isw2 X-ChIP-chip, Isw2 ChIPexo, and Isw2 remodeling) to generate average N-ChIP-seq profiles. We detected enrichment of Isw2 by N-ChIP-seq at sites bound by Isw2 in both X-ChIP-chip and ChIP-exo and sites of chromatin remodeling by Isw2 but not around randomly selected nucleosomes (Figure 2A). The two peaks on either side of the midpoint represent robust enrichment of Isw2 on either side of transcription factor binding sites. We note that the enrichment of
Isw2 at these sites does not imply an effect on expression of nearby genes, only that our technique is able to replicate Isw2 binding sites previously determined by X-ChIP methodologies.

Sites of Isw2 action display high A+T content, which stiffens DNA and disfavors nucleosome formation [32]. We analyzed the bendability of DNA at sites occupied solely by Isw2 or by Isw 1 and/or Chd1 without Isw2. Isw2-only N-ChIP-seq sites showed a reduction of $\sim 0.375$ in average bendability compared to Isw $1 /$ Chdl sites $\left(P=5.3 \times 10^{-41}\right)$. We also detected strong enrichment of Isw2 but little or no binding of Isw 1 or Chd1 at the A+T rich yeast recombination enhancer (Figure 2B), as previously demonstrated [32]. We further noted enrichment of Isw2 at the chromosome III centromere, a $\sim 90 \% \mathrm{~A}+\mathrm{T}$ sequence (Figure $2 \mathrm{G}$ ). These results indicate that our N-ChIP-seq protocol faithfully captures known features of Isw2 genomic association.

\section{Isw2 preferentially binds centromeres}

The enrichment of Isw2 at the chromosome III centromere led us to investigate Isw2 centromeric association in more detail. Aggregate analysis of remodeler signal at all 16 yeast centromeres revealed robust enrichment of Isw2, but not Isw1 or Chdl (Figure 3A). To precisely delineate the regions of the centromere bound by Isw2, we employed V-plotting [30]. In a V-plot, the midpoint of each paired-end fragment is assigned a dot in twodimensional space, wherein the $\mathrm{X}$-axis value is the distance of the fragment midpoint from a defined genomic feature and the $\mathrm{Y}$-axis value is the length of the fragment. V-plotting of Isw2 ChIP and input data revealed striking enrichment of Isw2 to the right of centromere midpoints, over the CDEII and CDEIII regions (Figure 3B). Analysis of centromeric sequence composition confirmed the $\mathrm{A}+\mathrm{T}$ rich nature of yeast centromeres, further supporting the preference of Isw2 for A+T rich DNA (Figure 3G). The association of Isw2 with centromeres is consistent with previous X-ChIP results showing association of Isw2 with two yeast centromeres [33] as well as studies demonstrating a role for Isw2 in pericentromeric nucleosome dynamics [34] and centromeric association of the human ISWI-containing RSF complex [35].

\section{ISWI and CHD remodelers occupy extended linker DNA at transcription factor binding sites}

Previous work has demonstrated that sequence-specific TFs induce nucleosome depletion upon binding [36-41], presumably by exposing stretches of linker DNA. We therefore hypothesized that the enrichment of supernucleosomal DNA fragments in ChIP samples might reflect remodeler association with the extended linker DNA flanking binding sites for nucleosome-phasing TFs. We assessed transcription factor binding site (TFBS) occupancy of Isw1, Isw2, and Chdl using V-plotting. We first analyzed remodeler occupancy at binding sites for the Abfl TF. Consistent with our previous data [30], well-phased flanking nucleosomes were observed at Abfl sites as discrete dot clusters to either side of the TFBS (Figure 4A and Figure S2A).

In samples treated with MNase for 2.5', we noted robust enrichment of supernucleosomal fragments spanning $\sim 250$ $300 \mathrm{bp}$ and centered between the TFBS and each flanking nucleosome in ChIP, and to a lesser extent, input samples. These supernucleosomal fragments represent continuous protection of DNA from the flanking nucleosome to the opposite side of the TFBS, with remodeler complexes occupying the linker DNA spanning the nucleosome and TFBS. Additionally, supernucleosomal fragments were depleted from the more heavily digested $10^{\prime}$ samples, while smaller fragments flanking both sides of the TFBS were seen (Figure 4A and Figure S2A), suggesting that remodelerlinker DNA associations display varying degrees of stability. 
A
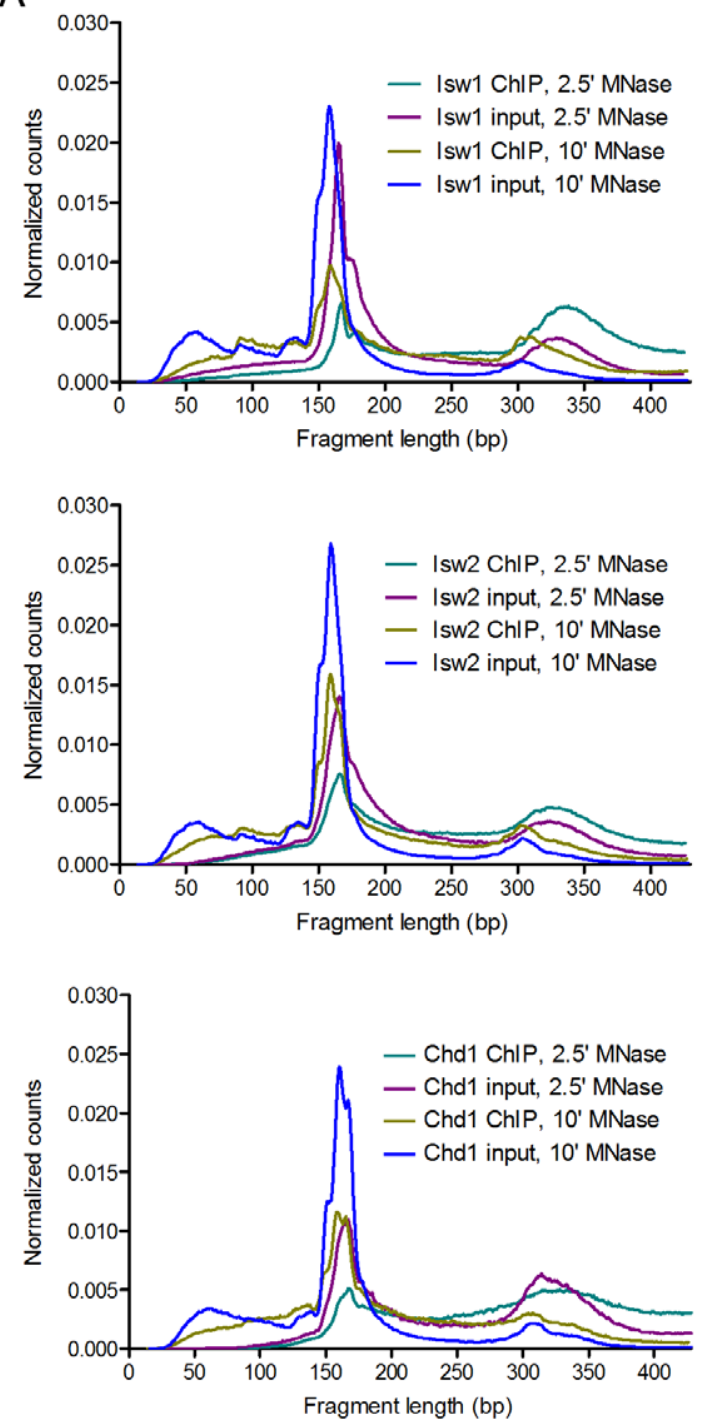

B
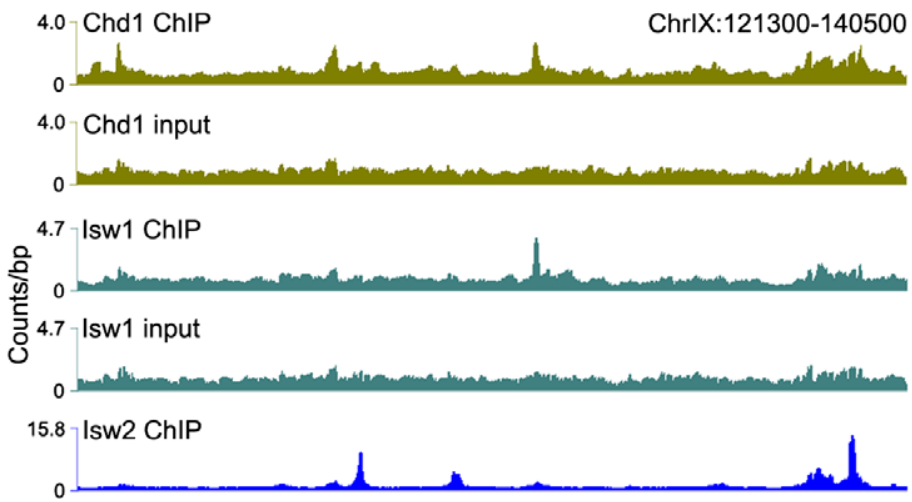

15.8 Isw2 input

Genes

$\sqrt{3}$

Figure 1. N-ChIP-seq localizes ISWI and CHD remodelers throughout the genome. (A) Size distributions of mapped paired-end 2.5' and 10' MNase-digested wild-type Isw1, Isw2, and Chd1 ChIP and input fragments. Slight variations in the amount of supernucleosomal (>251 bp) fragments are attributable to minor variation in the degree of MNase digestion for each sample, as evidenced by slight differences in the nucleosomal maxima for each sample. (B) Binding profiles for 10' MNase-digested Isw1, Isw2, and Chd1 samples across a representative region of the genome. The number of paired-end reads overlapping each genomic position (counts/bp) is indicated on the Y-axis. See Figure S1 for additional remodeler binding profiles. doi:10.1371/journal.pgen.1003317.g001

As observed previously, clusters of subnucleosomal particles flank TFBSs in our input chromatin samples [30]. These particles were not observed in remodeler ChIP V-plots (Figure $4 \mathrm{~A}$ and Figure S2A), indicating that they are not remodelers but rather small DNA-binding proteins. Fragment sizes in the central protected region of the ChIP V-plots extended $\sim 30$ bp higher than in the input $\mathrm{V}$-plots, perhaps indicating stable association of remodelers closer to the TFBS (Figure 4A and Figure S2A). We also generated V-plots of $2.5^{\prime}$ and $10^{\prime}$ MNase ChIP and input data using Cbfl, Mbpl, and Rebl binding sites, which, like Abfl sites, display well-phased flanking nucleosomes (Figure S2B-S2D). In each case, similar V-plots were seen for Isw1, Isw2, and Chd1.

These V-plot observations suggest that remodelers bind to regions of extended linker DNA created by TF-induced nucleosome depletion at TFBSs and protect variable amounts of flanking DNA (Figure 4B, top). A remodeler molecule then occupies an extended region up to the flanking nucleosome to generate the large supernucleosomal fragment clusters observed after $2.5^{\prime}$ MNase digestion. Unprotected linker DNA on the non-remodeler protected side of the TF could then be occupied by DNA-binding proteins (Figure 4B, bottom). Depletion of supernucleosomal fragments in the $10^{\prime}$ MNase-digested samples suggests that remodelers dynamically protect linker DNA on one or the other side of the TFBS. This association of Isw1, Isw2, and Chdl with substantial extranucleosomal DNA is consistent with in vitro studies in yeast, Drosophila, and humans [6-10,42,43].

\section{Remodeler association with transcription factor binding sites is ATP-independent}

We next asked whether remodeler inactivation would alter binding around TFBSs. Surprisingly, elimination of remodeler catalytic activity via a lysine-to-arginine substitution in the conserved GXGKT ATP-binding motif $[21,44]$ did not substantially affect the 
A

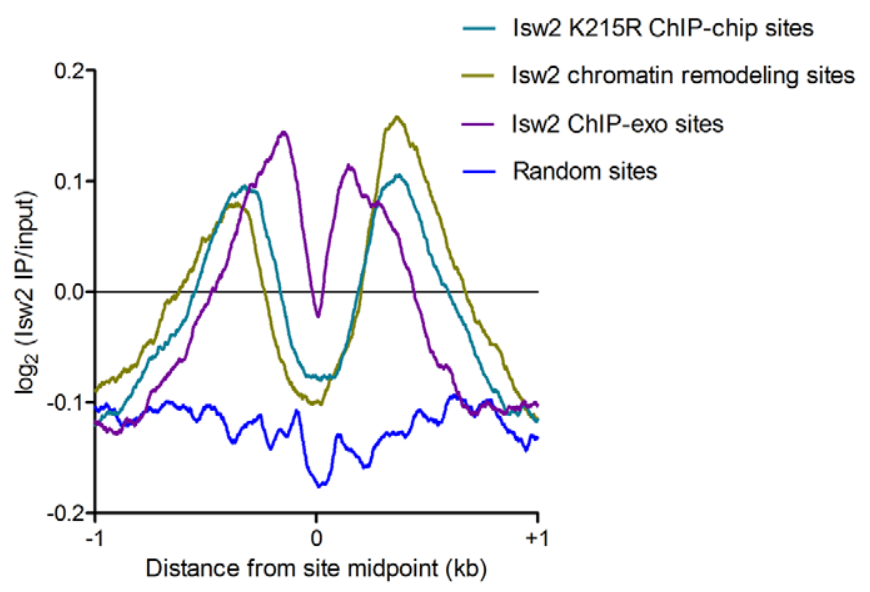

B

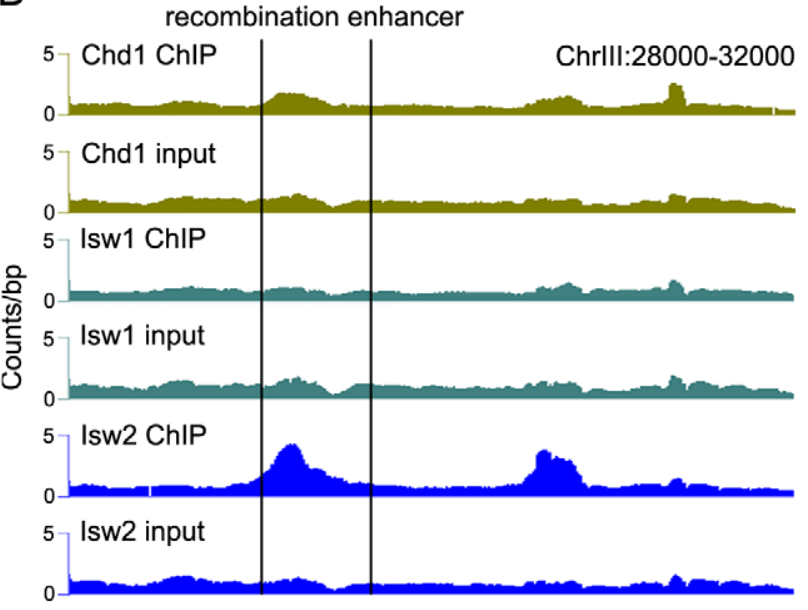

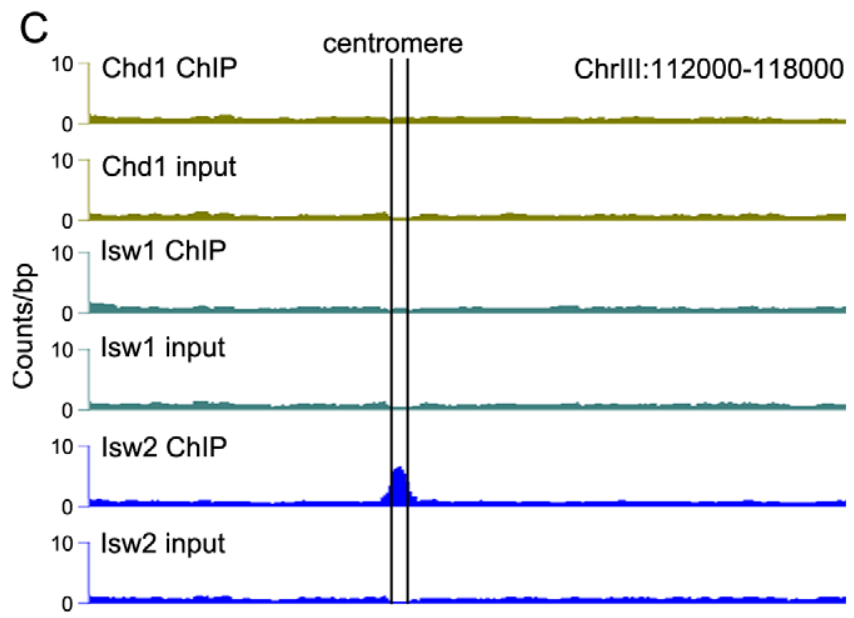

Figure 2. Comparison of Isw2 $\mathbf{N}$-ChIP and X-ChIP data. (A) Aggregate profiles of $\log _{2}(\mathrm{Isw} 2 \mathrm{IP} /$ input) at sites bound by catalytically inactive (K227R) Isw2 in ChIP-chip experiments (2128 sites), sites with altered nucleosome positioning in an isw24 strain (1399 sites), sites bound by wild-type Isw2 in ChIP-exo experiments (1251 sites), and around random nucleosomes (1399 sites).Also shown are profiles of remodeler binding at the (B) chrllI recombination enhancer and $(C)$ chrlll centromere (marked by vertical lines). doi:10.1371/journal.pgen.1003317.g002

size distribution of mapped fragments (Figure 5A and Figure S4) or the genomic binding profiles of remodelers (Figure 5B). We assessed the effect of remodeler catalytic inactivation by $\mathrm{V}$-plotting wild-type and catalytically inactive (K227R) ChIP and input data at sites for the Reb1 TF generated by ChIP-exo [45], which also allowed us to assess V-plot patterns using an independent set of binding sites. Vplot patterns generated with ChIP-exo Rebl sites were nearly indistinguishable from those created using Rebl ChIP-chip sites (Figure 5C, Figure S3, Figure S5). Catalytic inactivation of remodelers also had no noticeable effect on $\mathrm{V}$-plot patterns at TFBSs (Figure 5C, Figure S3, and Figure S5), implying that remodeler association with TFBSs is ATP-independent.

\section{ISWI and CHD remodelers preferentially bind NDRs}

The sharp patterns of phased nucleosomes on either side of binding sites for Abfl, Rebl and other TFs is consistent with their known role in creating NDRs that lead to transcriptional activation [46]. Iswland Chd1 position nucleosomes within gene bodies in vivo, while Isw2 generally positions nucleosomes flanking NDRs, preventing directional nucleosome shifting within gene bodies [16-21]. We therefore asked whether there is a relationship between remodeler occupancy and dynamics around individual TFBSs and features of adjacent genes. Consistent with their known functions, we observed enrichment of Chdl and Iswl within gene bodies (Figure 6A-6B), while Isw2 showed robust enrichment at NDRs, where TFBSs are generally located in yeast, with little gene body binding (Figure 6C). Strikingly, equal or slightly greater enrichment than that seen in gene bodies for Chdl and Iswl was observed at NDRs at the $5^{\prime}$ and $3^{\prime}$ ends of verified ORFs (Figure 6A-6B). We also detected Iswl association with $5^{\prime}$ NDRs after adding a formaldehyde crosslinking step to our N-ChIP-seq protocol (Figure S6), indicating that remodeler-NDR association is not due to opportunistic, nonspecific interactions of remodelers with free DNA during chromatin preparation and immunoprecipitation. We assessed the association of remodeler binding with NDR size, reasoning that if remodeler-NDR interactions were simply due to the presence of large regions of naked DNA in the chromatin preparation, larger NDRs would display greater remodeler association. No such correlations were observed (Iswl $R^{2}=0.0339$; Isw2 $R^{2}=0.0397$; Chd $1 R^{2}=0.0519$ ), further arguing against nonspecific association of remodelers with free DNA during our experiments.

Our Isw2 N-ChIP-seq results are somewhat different from those generated using ChIP-exo. We found Isw2 to be enriched upstream of ORF $5^{\prime}$ ends to a distance of nearly $1 \mathrm{~kb}$ in some cases 
A

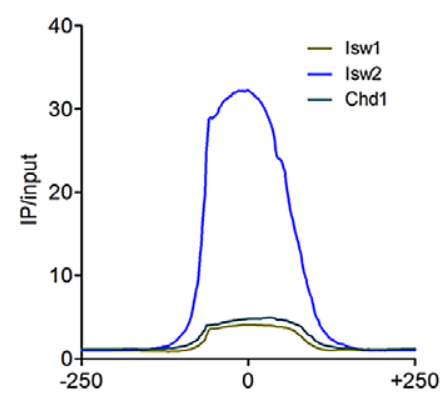

B
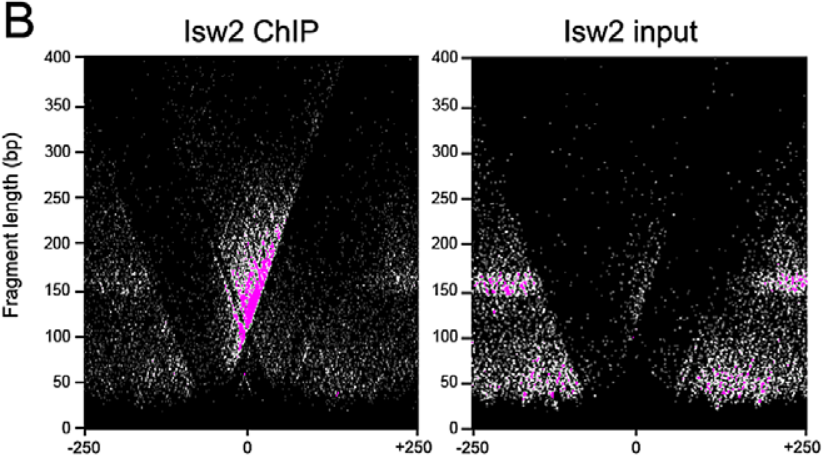

C

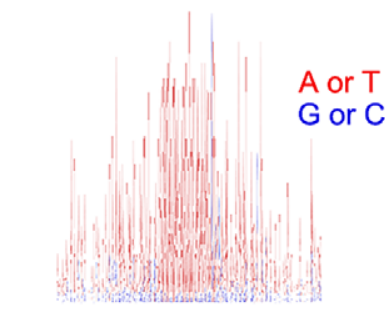

Distance from centromere midpoint $(\mathrm{bp})$

Figure 3. Isw2 associates with centromeres. (A) Aggregate plot of Isw1, Isw2, and Chd1 ChIP/input signal at all 16 yeast centromeres. (B) V-plot of Isw2 ChIP data for all 16 yeast centromeres showing enrichment of Isw2 to the CDEIII side of centromeres. (C) Sequence logo of all 16 yeast centromeres spanning $400 \mathrm{bp}$ centered on the centromere midpoint. A+T are represented as red and $\mathrm{G}+\mathrm{C}$ are represented as blue, demonstrating the high $A+T$ content of centromeres. The binding of Isw2 to centromeres is thus consistent with its preference for association with regions of high A+T content. The sequence logo was generated with WebLogo (http://weblogo. berkeley.edu).

doi:10.1371/journal.pgen.1003317.g003

(Figure 6C), whereas ChIP-exo showed very discrete localization of Isw2 to the TSS, immediately adjacent to the +1 nucleosome [17]. The differences between our N-ChIP-seq results and those of the ChIP-exo study may be explained by the crosslinking of Isw2 to the +1 nucleosome in ChIP-exo. Isw2 that is not adjacent to a nucleosome is less likely to be crosslinked and more likely to be lost during subsequent solubilization of chromatin. Our results indicate that there is at least some transient interaction between Isw2 and the +1 nucleosome, and we posit that this is the fraction of Isw2 previously mapped by ChIP-exo.

\section{ISWI and CHD remodeler association with gene bodies is associated with transcription-coupled nucleosome turnover}

Our results demonstrate that yeast ISWI and CHD remodelers associate not only with gene bodies, where their effects are seen, but also with regions of extended linker DNA flanking TFBSs within NDRs. In the case of Isw2, robust binding to linker DNA within NDRs is consistent both with its in vitro preference for substantial extranucleosomal DNA and its action positioning nucleosomes adjacent to NDRs [7-9,20]. However, while the binding of Chdl and Iswl to linker DNA within NDRs is in line with their in vitro association with significant amounts of linker DNA $[6,10]$, it appears at odds with their function within gene bodies [16-19,21]. To reconcile the robust occupancy of Isw1 and Chdl around NDRs with their function within gene bodies, we suggest that TFs generate regions of nucleosome depletion, which expose extended linker DNA and are thus favorable for remodeler-chromatin association. Transcriptional elongation then disrupts or evicts nucleosomes, extending regions of linker DNA within gene bodies and enabling efficient remodeling of the remaining gene-body nucleosomes and/or newly deposited nucleosomes by Isw1 and Chd1, while Isw2 simultaneously positions NDR-flanking nucleosomes to prevent directional shifting of gene-body nucleosomes, which could lead to harmful cryptic transcription [20].

This model predicts that Chdl and Iswl association with gene bodies depends on transcription rate. As such, we would not expect to observe an association between remodeler binding and steady-state expression levels, as infrequently transcribed genes may yield mRNAs with long half-lives and vice versa [47]. Indeed, we observed no correlation between remodeler occupancy and steady-state gene expression (Figure 7A). Given that transcription is associated with histone turnover [48-52], we hypothesized that there might be a relationship between remodeler association and turnover. High turnover within ORF $5^{\prime}$ ends was postulated to reflect a requirement to maintain nucleosome depletion at promoters [51], which is achieved, at least in part, by TFs such as Abfl and Rebl and the SWI/SNF-family remodeler RSC $[39,41]$. Comparison of histone turnover data with remodeler $\mathrm{N}$ ChIP-seq data revealed a positive association between binding of remodelers to ORF $5^{\prime}$ ends and gene bodies and nucleosome turnover (Figure 7B). We also compared remodeler binding with transcription rate and found that highly transcribed genes were more highly bound by Isw1, Isw2, and Chd1 (Figure 7C). Taken together, these data suggest that Iswl and Chdl binding within gene bodies displaying high nucleosome turnover is a consequence of transcriptional elongation. While Isw2 generally does not bind gene bodies, its loss affects gene body nucleosomes, which shift to their thermodynamically favored positions in its absence [20]. The increased binding of Isw2 to the $5^{\prime}$ NDRs of highly transcribed genes may therefore reflect the increased nucleosome disruption caused by transcriptional elongation and, consequently, the increased requirement for the positioning activity of Isw2.

What is the relationship of ISWI and CHD remodelers to transcription? Previous work has demonstrated that simultaneous deletion of Isw1, Isw2, and Chd1 in yeast does not alter promoter nucleosome occupancy or positioning [16], indicating that these remodelers are unlikely to influence RNA polymerase II binding to promoters. Strikingly, this same work showed that loss of Iswl, Isw2, and Chd 1 had relatively little effect on gene transcription, despite causing massive disorganization of gene body chromatin structure. These findings suggested that the major role of ISWI and CHD remodelers, and gene body chromatin organization in general, is to prevent cryptic transcription within gene bodies [20,53]. In the case of Chdl deletion, cryptic transcription is linked to increased gene body histone turnover, indicating a role for Chdl in suppressing histone turnover [53]. This result is also consistent with our finding that Chdl binding is associated with nucleosome turnover, as regions displaying high turnover would 


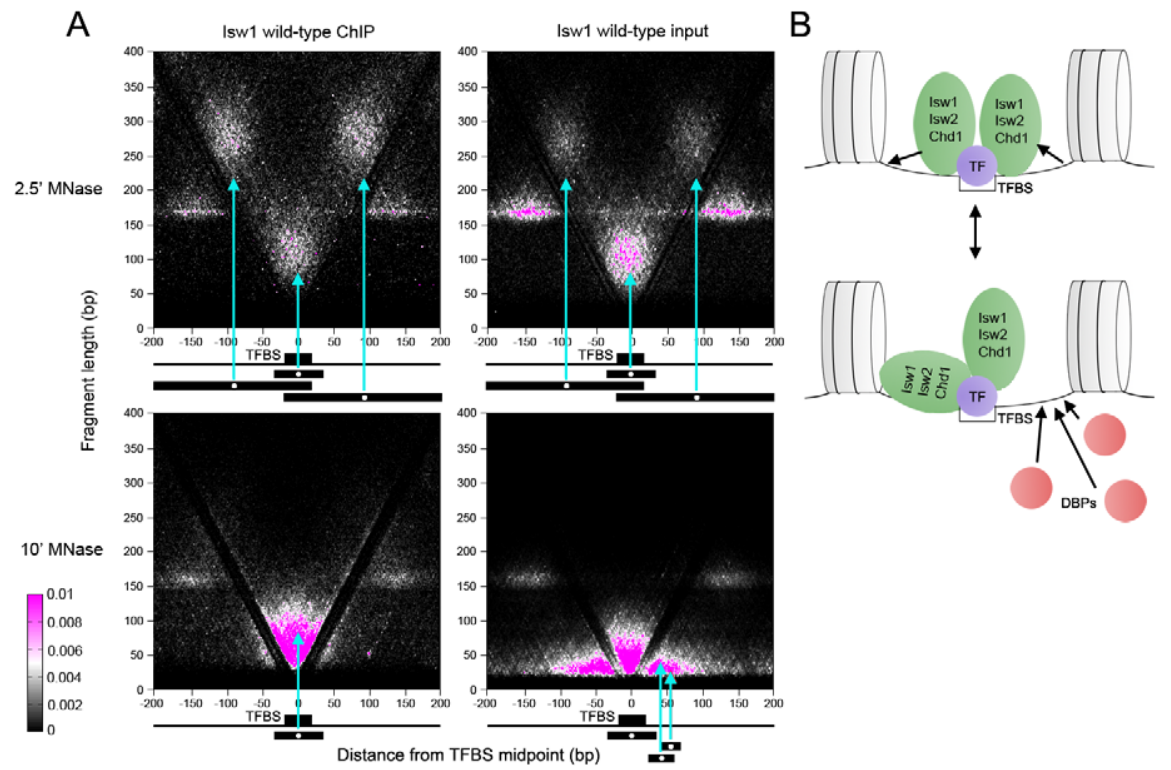

Figure 4. ISWI and CHD remodelers associate with TFBSs. (A) V-plots of wild-type Isw 1 ChIP and soluble input chromatin at binding sites for the Abf1 TF after 2.5' and 10' MNase digestion. Flanking nucleosomes are visualized as well-defined dot clusters on either side of the TFBS. Example fragments contributing to the generation of various V-plot features are shown schematically below each plot. Cyan arrows point to the position of each fragment midpoint within the V-plot. Similar results were seen for wild-type Isw2 and Chd1 at Abf1 sites and for Isw1, Isw2, and Chd1 at Cbf1, Mbp1, and Reb1 binding sites (Figures S3 and S4). (B) Interpretive schematic of V-plot results. DBPs; DNA-binding proteins.

doi:10.1371/journal.pgen.1003317.g004

both provide exposed DNA for Chdl to bind and require Chdl to suppress excessive histone turnover and subsequent cryptic transcription. Furthermore, loss of Chdl impairs post-elongation nucleosome reassembly [54]. In light of our data and these previous results, we propose the following model for the relationship of Chdl to transcription. A round of transcriptional
A

B
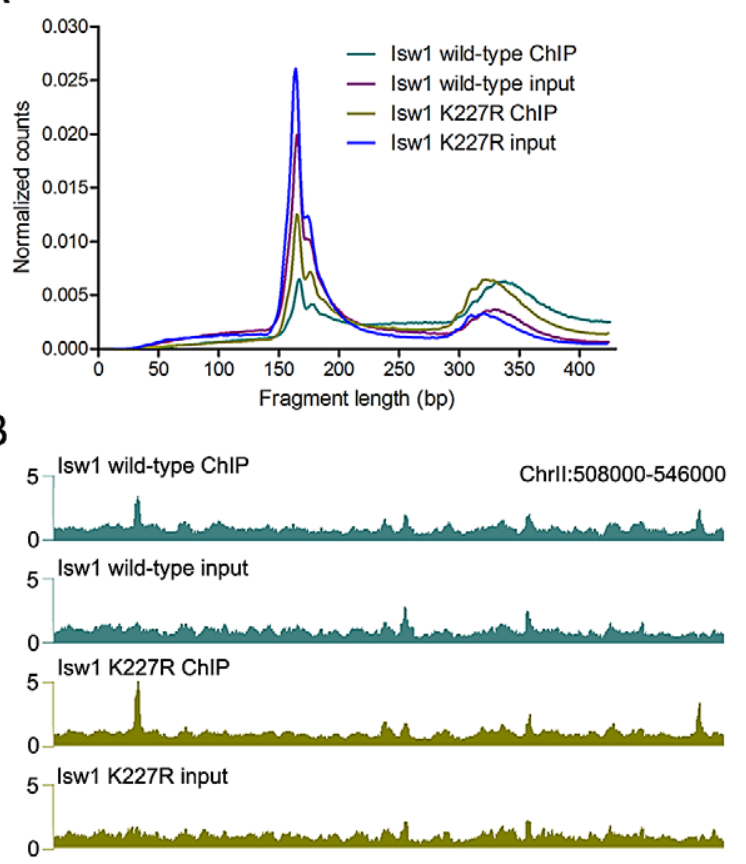

C
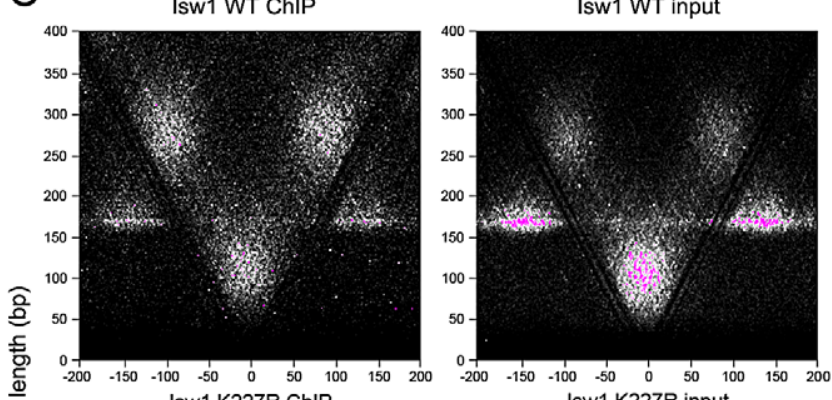

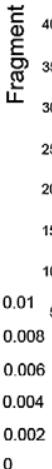

Isw1 K227R input

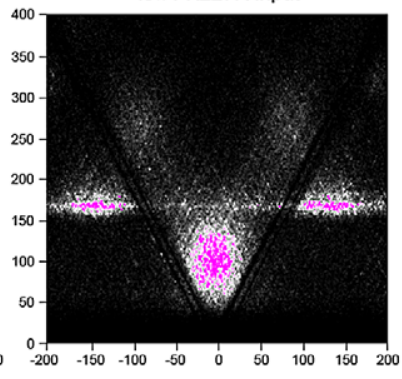

Distance from TFBS midpoint (bp)

Figure 5. ISWI and CHD remodeler association with TFBSs is ATP-independent. (A) Size distributions of mapped paired-end 2.5' MNasedigested wild-type and catalytically inactive K227R Isw1 ChIP and input fragments. Similar profiles were seen for Isw2 K215R and Chd1 K407R (Figure S4). (B) Profiles of wild-type and K227R Isw1 binding along a representative segment of the genome. (C) V-plots of wild-type and K227R Isw1 ChIP and soluble input chromatin at binding sites for the Reb1 TF, determined by ChIP-exo, after 2.5' MNase digestion. The overall fragment size in the Isw1 K227R ChIP and input samples is slightly reduced when compared to wild-type, indicative of technical variation in MNase digestion. Similar results were seen for K227R Isw1 and catalytically inactive Isw2 (K215R) and Chd1 (K407R) at Abf1 and other TFBSs (Figures S4 and S6).

doi:10.1371/journal.pgen.1003317.g005 

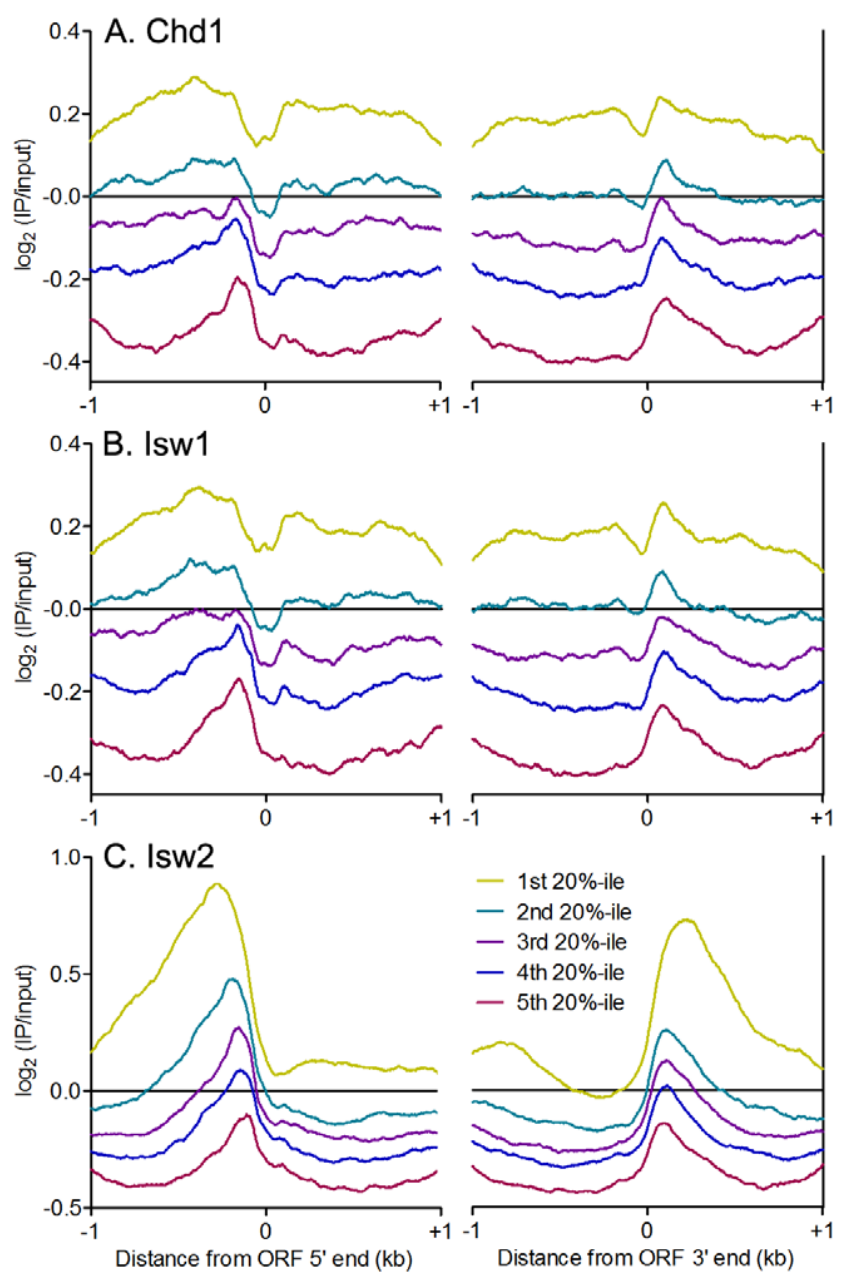

Figure 6. ISWI and CHD remodelers bind NDRs. Aggregate plots of $\log _{2}(\mathrm{IP} / \mathrm{input})$ signal $\pm 1 \mathrm{~kb}$ of verified ORF $5^{\prime}$ and $3^{\prime}$ ends for (A) Chd1, (B) Isw1, and (C) Isw2 ranked separated into quintiles by average remodeler signal.

doi:10.1371/journal.pgen.1003317.g006

initiation and elongation occurs, displacing or evicting nucleosomes. Chd1, either by interacting with the elongation machinery or following in the wake of RNA polymerase II, can then scan for regions of exposed linker DNA to which it can bind and suppress turnover and, by extension, cryptic transcription. In support of this possibility, Chdl has been shown to genetically and physically interact with the transcription machinery in several species [5558], and Isw1 and Isw2 genetically interact with elongation factors in yeast [59].

It is estimated that there is sufficient Isw2 for a molecule be bound every $2-5 \mathrm{~kb}$ of DNA, with a similar abundance for Chdl and twice that amount for Iswl (T.T., unpublished). Additionally, high salt is required for remodeler extraction from cells, indicating that they are bound to chromatin $[44,60]$. Therefore, as much as $5-10 \%$ of the yeast genome, perhaps most NDRs, may be occupied by Isw1, Isw2, and Chd1. Drosophila ISWI is similarly abundant [61] and multiple Chd1related remodelers are highly abundant and active in many higher eukaryotes [62-64], suggesting that the binding of ISWI and CHD remodelers at NDRs and their subsequent action downstream is an ancestral mechanism for chromatin structure maintenance.

\section{Materials and Methods}

\section{Yeast methods}

Yeast cells were grown in YPD and harvested at $\mathrm{OD}_{600}=0.6-$ 0.8. Mutation of Chd1 K407 was carried out as follows. Primers F5' - GGTACCATTGGGAGAATGCGACAGAT -3', containing a KpnI site (underlined) and R-5' - ATCGATCTTCGTCAACGGCGATAAAT - $3^{\prime}$, containing a ClaI site (underlined) were used to amplify nt 961-1543 of CHD1. The amplified fragment was TOPO-cloned into pCR2.1. pCR2.1-CHD1-9611543 was digested with $\mathrm{KpnI}$ and ClaI and the CHD1 fragment was ligated into pRS406 and mutagenized with the QuikChange kit (Agilent). Mutation was confirmed by sequencing and introduced a strain harboring a Chd1-3xFLAG allele via the pop-in/pop-out method [65]. Yeast strains used in this study are given in Table S1.

\section{Nuclear isolation and chromatin preparation}

Nuclei were isolated from yeast cells and stored at $-80^{\circ} \mathrm{C}$ in $5 \mathrm{ml}$ sorbitol-PIPES-calcium (SPG) buffer as described [66]. For $\mathrm{X}$-ChIP, cells were fixed with $1 / 10$ culture volume buffered formaldehyde solution (50 mM HEPES-KOH, $100 \mathrm{mM} \mathrm{NaCl}$, $1 \mathrm{mM}$ EDTA, $0.5 \mathrm{mM}$ EGTA, $11 \%$ formaldehyde) for $15 \mathrm{~min}$ at RT with gentle rotation and quenched with $18 \mathrm{ml} / 100 \mathrm{ml}$ culture volume $2.5 \mathrm{M}$ glycine prior to nuclear isolation. Upon thawing, SPC buffer was supplemented with $1 \mathrm{mM}$ phenylmethanesulfo-

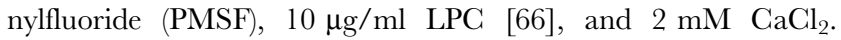
Chromatin was digested with MNase for 2.5 or $10 \mathrm{~min}$ and digestion was stopped by the addition of EDTA to $10 \mathrm{mM}$. MNase-treated nuclei were then passed 4 times through a 20gauge needle and 4 times through a 26-gauge needle [30,67] and centrifuged at 10,000 RPM for $10 \mathrm{~min}$. The supernatant (S1) was held on ice. The pellet was resuspended in $5 \mathrm{ml}$ extraction buffer (10 mM phosphate buffer $\mathrm{pH} 7.4,70 \mathrm{mM} \mathrm{NaCl}, 0.75 \mathrm{mM}$ EDTA, $0.1 \%$ Triton X-100) supplemented with $1 \mathrm{mM}$ PMSF and $10 \mu \mathrm{g} / \mathrm{ml}$ LPC and rocked on a nutator for 4 hours at $4^{\circ} \mathrm{C}$. Samples were centrifuged at 13,000 RPM for $15 \mathrm{~min}$ at $4^{\circ} \mathrm{C}$ and the supernatant (S2) was saved. Triton X-100 was added to S1 to a final concentration of $0.1 \%, \mathrm{~S} 1$ and $\mathrm{S} 2$ were combined, and the salt concentration of the combined $10 \mathrm{ml}$ chromatin solution was adjusted to $80 \mathrm{mM}$ with $\mathrm{NaCl}$.

\section{ChIP}

$100 \mu \mathrm{l}$ of each chromatin sample was saved as input. $100 \mu \mathrm{l}$ of FLAG M2 magnetic beads (Sigma M8823, lot \#041M6135) were used for all remodeler-FLAG immunoprecipitations (IPs). Beads were washed 3 times with $\mathrm{PBS} / 0.5 \%$ BSA and added to the chromatin samples. IPs were incubated overnight at $4{ }^{\circ} \mathrm{C}$ with endover-end rotation. Beads were washed 3 times with IP buffer (10 mM phosphate buffer, pH 7.4, $0.75 \mathrm{mM}$ EDTA, $70 \mathrm{mM}$ $\mathrm{NaCl}$ ) supplemented with $10 \mu \mathrm{g} / \mathrm{ml} \mathrm{LPC}$ and $1 \mathrm{mM}$ PMSF and resuspended in $400 \mu \mathrm{l} \mathrm{IP}$ buffer. $1 / 50^{\text {th }}$ volumes of $5 \mathrm{M} \mathrm{NaCl}$ and 0.5 mM EDTA were added to IP and input samples and RNA was digested with $1 \mu \mathrm{g}$ RNase A for $10 \mathrm{~min}$ at $37^{\circ} \mathrm{C}$. SDS was added to IP and input samples to a concentration of $0.5 \%$ and protein was digested with $80 \mu \mathrm{g}$ Proteinase $\mathrm{K}$ for $20 \mathrm{~min}$ at $65^{\circ} \mathrm{C}$. $20 \mu \mathrm{g}$ glycogen was added to each IP and input sample followed by extraction with 1 volume phenol/chloroform/isoamyl alcohol. DNA was precipitated with 2 volumes $100 \%$ ethanol for $30 \mathrm{~min}$ at $-80^{\circ} \mathrm{C}$ and washed twice with $500 \mu 100 \%$ ethanol. Precipitated DNA was resuspended in $20 \mu \mathrm{l} 0.1 \mathrm{X}$ TE buffer, $\mathrm{pH} 8.0$ and quantified by PicoGreen [30].

$\mathrm{X}$-ChIP was performed as above with the following modifications. In addition to washing in IP buffer, IPs were washed twice with TSE I $(0.1 \%$ SDS, $1 \%$ Triton X-100, 2 mM EDTA, $20 \mathrm{mM}$ 


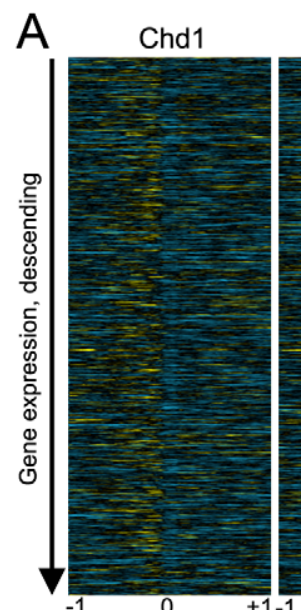

Isw1

Isw2

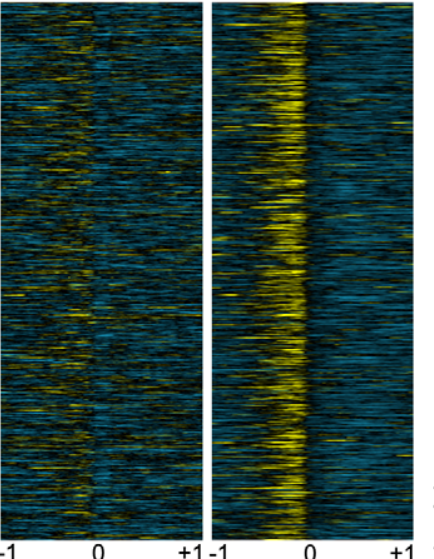

$\log _{2}$ (IP/input)

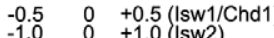

$\begin{array}{lll}-3.0 & 0 & +3.0\end{array}$

B Turnover

Chd1 Isw1

Isw2
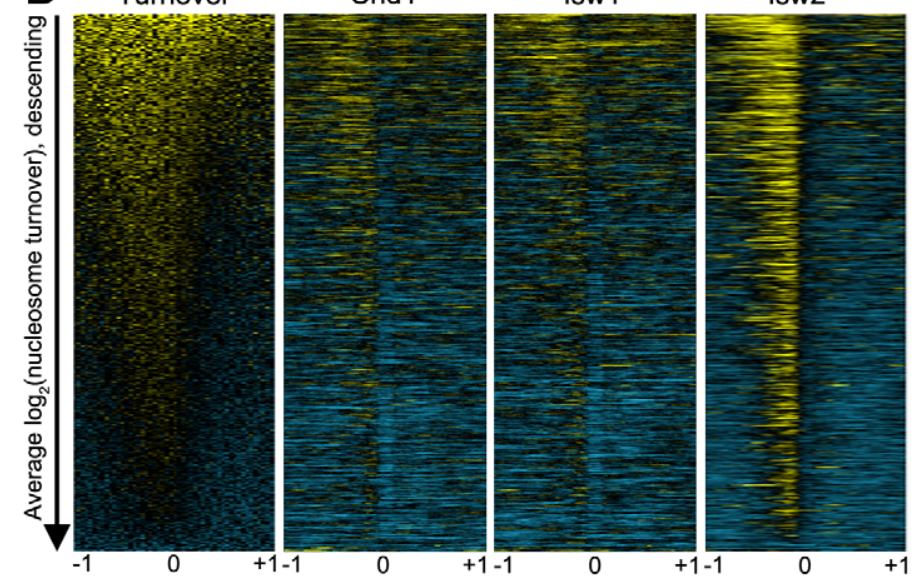

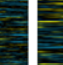
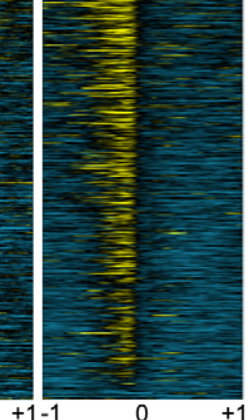

Distance from ORF 5' end (kb)

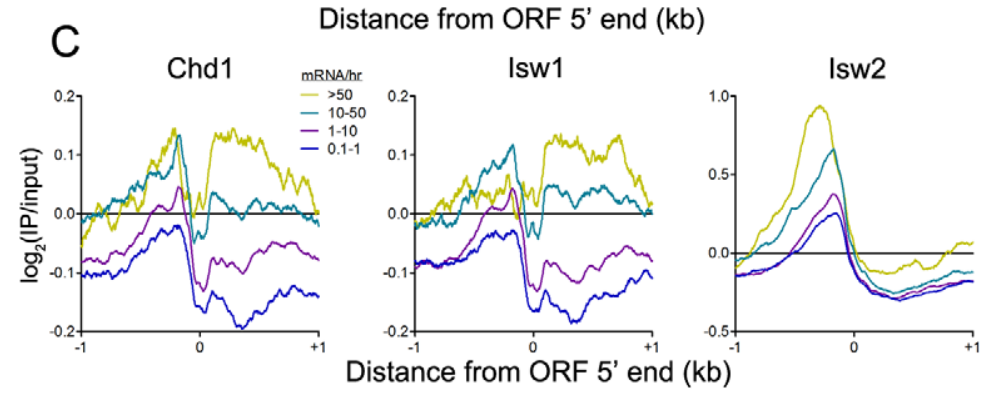

Figure 7. ISWI and CHD remodeler binding is positively associated with histone turnover and transcription rate. (A) Heat maps of $\log _{2}(I P /$ input) signal for Chd1, Isw1, and Isw2 $\pm 1 \mathrm{~kb}$ of verified ORF 5' ranked descending by gene expression. (B) Heat maps of log 2 (nucleosome turnover) and $\log _{2}\left(\mathrm{IP} /\right.$ input) signal for Chd1, Isw1, and Isw2 $\pm 1 \mathrm{~kb}$ of verified ORF $5^{\prime}$ ranked descending by average nucleosome turnover across the entire 2-kb window. (C) Aggregate plots of Chd1, Isw1, and Isw2 $\log _{2}(\mathrm{IP} /$ input) signal $\pm 1 \mathrm{~kb}$ of verified ORF 5 ' ends, separated by transcription rate in $\mathrm{mRNA} / \mathrm{hr}[47]$.

doi:10.1371/journal.pgen.1003317.g007

Tris $\mathrm{pH} 8.0,150 \mathrm{mM} \mathrm{NaCl})$ and twice with TSE II $(0.1 \% \mathrm{SDS}$, $1 \%$ Triton X-100, 2 mM EDTA, $20 \mathrm{mM}$ Tris $\mathrm{pH} 8.0,500 \mathrm{mM}$ $\mathrm{NaCl})$. Elution and crosslink reversal was performed overnight at $65^{\circ} \mathrm{C}$ in elution buffer $(50 \mathrm{mM}$ Tris $\mathrm{pH} 8.0,10 \mathrm{mM}$ EDTA, $1 \%$ SDS). Following elution, 1 volume of TE buffer $\mathrm{pH} 8.0$ was added to each sample to dilute the SDS to $0.5 \%$ for RNase A and proteinase $\mathrm{K}$ treatment as above.

\section{Sequencing library preparation}

Sequencing libraries were prepared according to our previously described modification of the standard Illumina protocol [30].
Libraries were sequenced for 25 cycles in paired-end mode on the Illumina HiSeq 2000 platform at the Fred Hutchinson Cancer Research Center Genomics Shared Resource.

\section{Data analysis}

Paired-end sequencing data were processed and aligned to the V64/SacCer3 genome build with Novoalign (Novocraft; http:// www.novocraft.com) and processed as described [30,68]. Numbers of paired-end fragments mapped to the genome for each sample are given in Table S2. Size distributions were normalized such that the area under the curve for each sample was equal to 1 . V-plots 
were generated with gnuplot (http://www.gnuplot.info). The Vplot scale represents the percentage of fragments used in constructing each V-plot contained in each bin (pixel) of the plot (160,400 bins total).

Isw2 K215R ChIP-chip binding sites, sites of chromatin remodeling by Isw2, and random sites were obtained from Whitehouse et al. [20] and Isw2 ChIP-exo binding sites were obtained from Yen et al. [17]. The $\log _{2}(\mathrm{IP} /$ input $)$ N-ChIP-seq signal for each base pair $\pm 1 \mathrm{~kb}$ of the midpoint of each Isw2 K215R-bound region, site of Isw2 remodeling, Isw2 ChIP-exo site, and random site was determined as above and averaged to generate aggregate plots.

For DNA bendability analysis, $29100 \mathrm{bp}$ regions bound by Isw2 without Isw1 and/or Chd1 and 29100 bp regions bound by Isw1 and/or Chd1 without Isw2 were obtained. Regions were analyzed using the bend.it server [69] with a window size of 39 bp. Bendability values for each position within the window were averaged and the two classes of sites were compared by t-test.

The SGD verified ORFs list was used to determine transcription unit length and ORF $5^{\prime}$ and $3^{\prime}$ end coordinates. For heatmaps, the $\log _{2}$ (IP/input) for each base pair $\pm 1 \mathrm{~kb}$ of each ORF $5^{\prime}$ or $3^{\prime}$ coordinate was determined using a custom Perl script [70]. Heatmaps were visualized with Java TreeView [71]. All heatmaps were generated using $10^{\prime}$ MNase data and are oriented such that the direction of transcription for all genes is to the right. Expression data were obtained from GEO (GSM552681) [72]. Nucleosome turnover data were obtained from Dion et al. [51]. Nucleosome turnover signal was obtained as above, but using $40 \mathrm{bp}$ windows due to the lower resolution of these data. Transcription rates were obtained from Holstege et al. [47]. Correlations between remodeler signal and NDR size were generated using a previously annotated list of NDRs [73] and the maximum $\log _{2}$ (IP/input) N-ChIP-seq signal within each NDR.

\section{Data availability}

Sequencing data generated in this publication have been deposited with GEO (GSE39331).

\section{Supporting Information}

Figure S1 Additional Isw1, Isw2, and Chd1 N-ChIP-seq profiles. Signal tracks of Isw1, Isw2, and Chd1 binding in representative regions of the genome. Counts/bp is indicated on the $\mathrm{Y}$-axis.

(TIF)

\section{References}

1. Flaus A, Martin DMA, Barton GJ, Owen-Hughes T (2006) Identification of multiple distinct Snf2 subfamilies with conserved structural motifs. Nucleic Acids Res 34: 2887-2905.

2. Wilson BG, Roberts CWM (2011) SWI/SNF nucleosome remodellers and cancer. Nat Rev Cancer 11: 481-492.

3. Ryan RJH, Bernstein BE (2012) Genetic Events That Shape the Cancer Epigenome. Science 336: 1513-1514.

4. Boerkoel CF, Takashima H, John J, Yan J, Stankiewicz P, et al. (2002) Mutant chromatin remodeling protein SMARCAL1 causes Schimke immuno-osseous dysplasia. Nat Genet 30: 215-220.

5. Zentner GE, Layman WS, Martin DM, Scacheri PC (2010) Molecular and phenotypic aspects of CHD7 mutation in CHARGE syndrome. Am J Med Genet A 152A: 674-686.

6. Gangaraju VK, Bartholomew B (2007) Dependency of ISW1a Chromatin Remodeling on Extranucleosomal DNA. Mol Cell Biol 27: 3217-3225.

7. Zofall M, Persinger J, Bartholomew B (2004) Functional Role of Extranucleosomal DNA and the Entry Site of the Nucleosome in Chromatin Remodeling by ISW2. Mol Cell Biol 24: 10047-10057.

8. Dang W, Kagalwala MN, Bartholomew B (2007) The Dpb4 Subunit of ISW2 Is Anchored to Extranucleosomal DNA. J Biol Chem 282: 19418-19425.
Figure S2 V-plots of wild-type Isw1, Isw2 and Chd1 ChIP and input data at Abfl, Cbfl, Mbpl, and Rebl sites. Note the discrete lower size limit of flanking nucleosomes at $\sim 147 \mathrm{bp}$ in $10^{\prime}$ MNase-treated samples, indicating that these nucleosomes are tightly wrapped.

(TIF)

Figure S3 V-plots of wild-type and catalytically inactive Isw1, Isw2, and Chdl ChIP and input data at ChIP-exo-defined Rebl binding sites. Binding sites are derived from the data of Rhee and Pugh [45].

(TIF)

Figure S4 Loss of remodeler catalytic activity does not alter remodeler fragment size distributions. Size distributions of mapped paired-end 2.5' MNase-digested wild-type and K215R Isw2 and wild-type and K407R Chd1 ChIP and input fragments. (TIF)

Figure S5 V-plots of catalytically inactive Isw1, Isw2 and Chd1 ChIP and input data at Abfl, Cbfl, Mbpl, and Rebl sites.

(TIF)

Figure S6 Iswl association with $5^{\prime} \mathrm{NDRs}$ is captured by $\mathrm{X}$ ChIP-seq. Heatmaps of $\log _{2}$ (Isw1 IP/input) native and crosslinked $2.5^{\prime}$ MNase signal $\pm 1 \mathrm{~kb}$ of verified ORF $5^{\prime}$ ends ranked descending by gene expression level.

(TIF)

Table S1 Yeast strains used in this study. All strains are derived from W1588-4C, which is isogenic to W303-1A except that a weak RAD5 mutation is repaired [74].

(DOC)

Table S2 Numbers of mapped pairs and supernucleosomal/ nucleosomal AUC ratios for sequenced samples. ChIP/input is the ChIP supernucleosomal/nucleosomal AUG ratio divided by the input supernucleosomal/nucleosomal AUC ratio.

(DOG)

\section{Acknowledgments}

We thank Jorja Henikoff for assistance with data analysis and Sheila Teves and Siva Kasinathan for comments on the manuscript.

\section{Author Contributions}

Conceived and designed the experiments: GEZ TT SH. Performed the experiments: GEZ. Analyzed the data: GEZ SH. Contributed reagents/ materials/analysis tools: GEZ TT SH. Wrote the paper: GEZ TT SH.

9. Kagalwala MN, Glaus BJ, Dang W, Zofall M, Bartholomew B (2004) Topography of the ISW2-nucleosome complex: insights into nucleosome spacing and chromatin remodeling. EMBO J 23: 2092-2104.

10. McKnight JN, Jenkins KR, Nodelman IM, Escobar T, Bowman GD (2011) Extranucleosomal DNA Binding Directs Nucleosome Sliding by Chd1. Mol Cell Biol 31: 4746-4759.

11. Gossett AJ, Lieb JD (2012) In Vivo Effects of Histone H3 Depletion on Nucleosome Occupancy and Position in Saccharomyces cerevisiae. PLoS Genet 8: e1002771. doi:10.1371/journal.pgen.1002771

12. Racki LR, Yang JG, Naber N, Partensky PD, Acevedo A, et al. (2009) The chromatin remodeller ACF acts as a dimeric motor to space nucleosomes. Nature 462: 1016-1021.

13. Yang JG, Madrid TS, Sevastopoulos E, Narlikar GJ (2006) The chromatinremodeling enzyme ACF is an ATP-dependent DNA length sensor that regulates nucleosome spacing. Nat Struct Mol Biol 13: 1078-1083.

14. Blosser TR, Yang JG, Stone MD, Narlikar GJ, Zhuang X (2009) Dynamics of nucleosome remodelling by individual ACF complexes. Nature 462: 1022-1027.

15. Bouazoune K, Kingston RE (2012) Chromatin remodeling by the CHD7 protein is impaired by mutations that cause human developmental disorders. Proc Natl Acad Sci U S A. 
16. Gkikopoulos T, Schofield P, Singh V, Pinskaya M, Mellor J, et al. (2011) A Role for Snf2-Related Nucleosome-Spacing Enzymes in Genome-Wide Nucleosome Organization. Science 333: 1758-1760.

17. Yen K, Vinayachandran V, Batta K, Koerber RT, Pugh BF (2012) Genomewide Nucleosome Specificity and Directionality of Chromatin Remodelers. Cell 149: 1461-1473.

18. Xella B, Goding C, Agricola E, Di Mauro E, Caserta M (2006) The ISWI and CHD1 chromatin remodelling activities influence ADH2 expression and chromatin organization. Mol Microbiol 59: 1531-1541.

19. Tirosh I, Sigal N, Barkai N (2010) Widespread remodeling of mid-coding sequence nucleosomes by Isw1. Genome Biol 11: R49.

20. Whitehouse I, Rando OJ, Delrow J, Tsukiyama T (2007) Chromatin remodelling at promoters suppresses antisense transcription. Nature 450: $1031-1035$.

21. Simic R, Lindstrom DL, Tran HG, Roinick KL, Costa PJ, et al. (2003) Chromatin remodeling protein Chdl interacts with transcription elongation factors and localizes to transcribed genes. EMBO J 22: 1846-1856.

22. Albert I, Mavrich TN, Tomsho LP, Qi J, Zanton SJ, et al. (2007) Translational and rotational settings of H2A.Z nucleosomes across the Saccharomycescerevisiae genome. Nature 446: 572-576.

23. Shim YS, Choi Y, Kang K, Cho K, Oh S, et al. (2012) Hrp3 controls nucleosome positioning to suppress non-coding transcription in eu- and heterochromatin. EMBO J 31: 4375-4387.

24. Pointner J, Persson J, Prasad P, Norman-Axelsson U, Stralfors A, et al. (2012) CHD1 remodelers regulate nucleosome spacing in vitro and align nucleosomal arrays over gene coding regions in S. pombe. EMBO J 31: 4388-4403.

25. Hennig BP, Bendrin K, Zhou Y, Fischer T (2012) Chdl chromatin remodelers maintain nucleosome organization and repress cryptic transcription. EMBO Rep 13: 997-1003.

26. Lantermann AB, Straub T, Stralfors A, Yuan G-C, Ekwall K, et al. (2010) Schizosaccharomyces pombe genome-wide nucleosome mapping reveals positioning mechanisms distinct from those of Saccharomyces cerevisiae. Nat Struct Mol Biol 17: 251-257.

27. O'Neill LP, Turner BM (2003) Immunoprecipitation of native chromatin: NChIP. Methods 31: 76-82.

28. Roca H, Franceschi RT (2008) Analysis of transcription factor interactions in osteoblasts using competitive chromatin immunoprecipitation. Nucleic Acids Res 36: 1723-1730.

29. Kent NA, Adams S, Moorhouse A, Paszkiewicz K (2011) Chromatin particle spectrum analysis: a method for comparative chromatin structure analysis using paired-end mode next-generation DNA sequencing. Nucleic Acids Res 39: e26.

30. Henikoff JG, Belsky JA, Krassovsky K, MacAlpine DM, Henikoff S (2011) Epigenome characterization at single base-pair resolution. Proc Natl Acad Sci U S A 108: 18318-18323.

31. Gelbart ME, Bachman N, Delrow J, Boeke JD, Tsukiyama T (2005) Genomewide identification of Isw2 chromatin-remodeling targets by localization of a catalytically inactive mutant. Genes Dev 19: 942-954.

32. Whitehouse I, Tsukiyama T (2006) Antagonistic forces that position nucleosomes in vivo. Nat Struct Mol Biol 13: 633-640.

33. Zhang Z, Reese JC (2004) Ssn6-Tup1 requires the ISW2 complex to position nucleosomes in Saccharomyces cerevisiae. EMBO J 23: 2246.

34. Verdaasdonk JS, Gardner R, Stephens AD, Yeh E, Bloom K (2012) Tensiondependent nucleosome remodeling at the pericentromere in yeast. Mol Biol Cell 23: 2560-2570.

35. Perpelescu M, Nozaki N, Obuse C, Yang H, Yoda K (2009) Active establishment of centromeric CENP-A chromatin by RSF complex. J Cell Biol 185: 397-407.

36. Tsukiyama T, Becker PB, Wu C (1994) ATP-dependent nucleosome disruption at a heat-shock promoter mediated by binding of GAGA transcription factor. Nature 367: 525-532.

37. Bernstein B, Liu C, Humphrey E, Perlstein E, Schreiber S (2004) Global nucleosome occupancy in yeast. Genome Biol 5: R62.

38. Ganapathi M, Palumbo MJ, Ansari SA, He Q Tsui K, et al. (2011) Extensive role of the general regulatory factors, Abfl and Rapl, in determining genomewide chromatin structure in budding yeast. Nucleic Acids Res 39: 2032-2044.

39. Bai L, Ondracka A, Cross Frederick R (2011) Multiple Sequence-Specific Factors Generate the Nucleosome-Depleted Region on CLN2 Promoter. Mol Cell 42: 465-476.

40. Lickwar GR, Mueller F, Hanlon SE, McNally JG, Lieb JD (2012) Genome-wide protein-DNA binding dynamics suggest a molecular clutch for transcription factor function. Nature 484: 251-255.

41. Hartley PD, Madhani HD (2009) Mechanisms that Specify Promoter Nucleosome Location and Identity. Cell 137: 445-458.

42. Brehm A, Langst G, Kehle J, Clapier CR, Imhof A, et al. (2000) dMi-2 and ISWI chromatin remodelling factors have distinct nucleosome binding and mobilization properties. EMBO J 19: 4332-4341.

43. Yang JG, Madrid TS, Sevastopoulos E, Narlikar GJ (2006) The chromatinremodeling enzyme ACF is an ATP-dependent DNA length sensor that regulates nucleosome spacing. Nat Struct Mol Biol 13: 1078-1083.
44. Tsukiyama T, Palmer J, Landel CC, Shiloach J, Wu C (1999) Characterization of the Imitation Switch subfamily of ATP-dependent chromatin-remodeling factors in Saccharomyces cerevisiae. Genes Dev 13: 686-697.

45. Rhee HS, Pugh BF (2011) Comprehensive Genome-wide Protein-DNA Interactions Detected at Single-Nucleotide Resolution. Cell 147: 1408-1419.

46. Iyer VR (2012) Nucleosome positioning: bringing order to the eukaryotic genome. Trends Cell Biol 22: 250-256.

47. Holstege FCP, Jennings EG, Wyrick JJ, Lee TI, Hengartner CJ, et al. (1998) Dissecting the Regulatory Circuitry of a Eukaryotic Genome. Cell 95: 717-728.

48. Schwabish MA, Struhl K (2004) Evidence for Eviction and Rapid Deposition of Histones upon Transcriptional Elongation by RNA Polymerase II. Mol Cell Biol 24: 10111-10117.

49. Kristjuhan A, Svejstrup JQ (2004) Evidence for distinct mechanisms facilitating transcript elongation through chromatin in vivo. EMBO J 23: 4243-4252.

50. Workman JL (2006) Nucleosome displacement in transcription. Genes Dev 20: 2009-2017.

51. Dion MF, Kaplan T, Kim M, Buratowski S, Friedman N, et al. (2007) Dynamics of Replication-Independent Histone Turnover in Budding Yeast. Science 315: 1405-1408.

52. Mito Y, Henikoff JG, Henikoff S (2005) Genome-scale profiling of histone H3.3 replacement patterns. Nat Genet 37: 1090-1097.

53. Smolle M, Venkatesh S, Gogol MM, Li H, Zhang Y, et al. (2012) Chromatin remodelers Isw 1 and Chd 1 maintain chromatin structure during transcription by preventing histone exchange. Nat Struct Mol Biol 19: 884-892.

54. Lee J-S, Garrett AS, Yen K, Takahashi Y-H, Hu D, et al. (2012) Codependency of H2B monoubiquitination and nucleosome reassembly on Chd1. Genes Dev 26: 914-919.

55. Kelley DE, Stokes DG, Perry RP (1999) CHD1 interacts with SSRP1 and depends on both its chromodomain and its ATPase/helicase-like domain for proper association with chromatin. Chromosoma 108: 10-25.

56. Simic R, Lindstrom DL, Tran HG, Roinick KL, Costa PJ, et al. (2003) Chromatin remodeling protein Chdl interacts with transcription elongation factors and localizes to transcribed genes. EMBO J 22: 1846-1856.

57. Quan TK, Hartzog GA (2010) Histone H3K4 and K36 Methylation, Chd1 and Rpd3S Oppose the Functions of Saccharomyces cerevisiae Spt4-Spt5 in Transcription. Genetics 184: 321-334.

58. Lin JJ, Lehmann LW, Bonora G, Sridharan R, Vashisht AA, et al. (2011) Mediator coordinates PIC assembly with recruitment of CHD1. Genes Dev 25: 2198-2209.

59. Collins SR, Miller KM, Maas NL, Roguev A, Fillingham J, et al. (2007) Functional dissection of protein complexes involved in yeast chromosome biology using a genetic interaction map. Nature 446: 806-810.

60. Tran HG, Steger DJ, Iyer VR, Johnson AD (2000) The chromo domain protein Chdlp from budding yeast is an ATP-dependent chromatin-modifying factor. EMBO J 19: 2323-2331.

61. Tsukiyama T, Daniel C, Tamkun J, Wu C (1995) ISWI, a member of the SWl2/ SNF2 ATPase family, encodes the $140 \mathrm{kDa}$ subunit of the nucleosome remodeling factor. Cell 83: 1021-1026.

62. Wang M, Weiss M, Simonovic M, Haertinger G, Schrimpf SP, et al. (2012) $\mathrm{PaxDb}$, a database of protein abundance averages across all three domains of life. Mol Cell Proteomics.

63. Hall JA, Georgel PT (2007) CHD proteins: a diverse family with strong ties. Biochem Cell Biol 85: 463-476.

64. Marfella CGA, Imbalzano AN (2007) The Chd family of chromatin remodelers. Mutat Res 618: 30-40.

65. Scherer S, Davis R (1979) Replacement of chromosome segments with altered DNA sequences constructed in vitro. Proc Natl Acad Sci U S A 76: 4951-4955.

66. Furuyama S, Biggins S (2007) Centromere identity is specified by a single centromeric nucleosome in budding yeast. Proc Natl Acad Sci U S A 104: 14706-14711.

67. Jin C, Felsenfeld G (2007) Nucleosome stability mediated by histone variants H3.3 and H2A.Z. Genes Dev 21: 1519-1529.

68. Krassovsky K, Henikoff JG, Henikoff S (2012) Tripartite organization of centromeric chromatin in budding yeast. Proc Natl Acad Sci U S A 109: $243-$ 248.

69. Vlahoviček K, Kaján L, Pongor S (2003) DNA analysis servers: plot.it, bend.it, model.it and IS. Nucleic Acids Res 31: 3686-3687.

70. Zentner GE, Saiakhova A, Manaenkov P, Adams MD, Scacheri PC (2011) Integrative genomic analysis of human ribosomal DNA. Nucleic Acids Res 39: 4949-4960.

71. Saldanha AJ (2004) Java Treeview - extensible visualization of microarray data. Bioinformatics 20: 3246-3248.

72. Tsankov AM, Thompson DA, Socha A, Regev A, Rando OJ (2010) The Role of Nucleosome Positioning in the Evolution of Gene Regulation. PLoS Biol 8: e1000414. doi:10.1371/journal.pbio.1000414

73. Yadon AN, Van de Mark D, Basom R, Delrow J, Whitehouse I, et al. (2010) Chromatin Remodeling around Nucleosome-Free Regions Leads to Repression of Noncoding RNA Transcription. Mol Cell Biol 30: 5110-5122.

74. Zhao X, Muller EGD, Rothstein R (1998) A Suppressor of Two Essentia Checkpoint Genes Identifies a Novel Protein that Negatively Affects dNTP Pools. Mol Cell 2: 329-340. 